\title{
Cytotoxic chemotherapy in advanced non-small cell lung cancer
}

\author{
G.V. Scagliotti, S. Novello, G. Selvaggi
}

Monaldi Arch Chest Dis 2005; 63: 4, 181-183.

University of Turin, Department of Clinical \& Biological Sciences, S. Luigi Hospital, Thoracic Oncology Unit, Orbassano (Torino), Italy.

Correspondence: Prof. Giorgio V. Scagliotti, MD, PhD, University of Turin, Department of Clinical \& Biological Sciences; S. Luigi Hospital, Thoracic Oncology Unit; Regione Gonzole, 10; 10043 Orbassano (Torino), Italy.

Lung cancer is still the leading cause of cancer-related deaths in Western countries [1]. In the past decade incidence rates showed a decreasing trend in men, while is on the rise in women. The 5year survival rate in the 1990 s was $14 \%$, which represents a minimal increase from the historical $13 \%$ of the 1970s. Around $80 \%$ of cases are NonSmall Cell Lung Cancer (NSCLC) and most of patients present with either locally advanced (stage III) or metastatic disease (stage IV).

The cure rate for radically resected patients does not exceed $70 \%$ in stage I disease, thus creating the issue of occult micro-metastatic disease at diagnosis. Therefore almost all patients will need at some point of their clinical history a systemic treatment to control the spread of the disease. In advanced NSCLC chemotherapy remains the cornerstone of treatment. A crucial step is whether the impact of chemotherapy in the management of this disease justifies either the toxicity and the ever increasing costs. Differing points of view are still quite common among medical and radiation oncologists, respiratory physicians and thoracic surgeons, which may ultimately lead to different clinical approaches.

Historically, chemotherapy in advanced stages has been viewed as palliative [2]. A survey from the U.S. showed how less than one-third of pulmunologists had some belief in the curative effect of chemotherapy in advanced NSCLC [3]. In the past only one-fifth of patients with metastatic lung cancer routinely received any form of treatment outside clinical trials. No clear advantage from agents in use at the time was evident, with response rates of less than $20 \%$ with single-agent cisplatin, mitomycin, ifosfamide, etoposide, and the vinca alkaloids vinblastine and vindesine; results were slightly higher for combination regimens but without any significant impact on longterm survival.

However a number of randomised trials demonstrated an improved 1-year survival rate for patients treated with chemotherapy when compared with BSC. This data was then grouped into a meta-analysis [4]: 11 trials examined BSC versus chemotherapy plus BSC (8 out of 11 trials used cisplatin-based regimens) in stage III and IV NSCLC. Patients treated with cisplatin-containing regimens demonstrated a $27 \%$ reduction in the risk of death, which translated into an absolute improvement in survival of $10 \%$ at 1 year with a modest but significant gain in median survival of 1.5 months. Further analysis did not demonstrate that any subgroup based on sex, age, histology, performance status, or stage could benefit more or less than others. Evidence that patients who received cisplatin-based chemotherapy in addition to surgery or radiotherapy also had improved outcomes strongly supported the idea that cisplatin plays a major role in combined modality treatment of NSCLC. The majority of combinations consisted of cisplatin plus either a vinca alkaloid or etoposide: although results were modest, they were nonetheless important from a public health perspective, given the large numbers of patients with NSCLC who could potentially receive benefits from such treatment.

The optimistic view is further supported by one of the first prospective studies designed to measure the impact of chemotherapy on quality of life that showed an improvement of tumor-related symptoms in $69 \%$ of patients: efficacy was highest in the first 2 courses thus showing an early palliation [5]. Two further randomised studies confirmed how quality of life, as measured through specific questionnaires, definitely improved in the chemotherapy arm [6,7]. Moreover with an eye toward health economics, chemotherapy proved superior over BSC in terms of cost-effectiveness due to a reduced number of days of hospitalisation as a result of a better control of cancer-related symptoms [8]. This data could also be reproduced by analysing costs from other studies employing cisplatin-based combinations with either vinorelbine or gemcitabine $[9,10,11]$.

In the 1990s new agents with novel mechanisms of action and a proven activity in NSCLC were introduced into common clinical practice. Such agents include the taxanes (paclitaxel and docetaxel), a new vinca alkaloid (vinorelbine), a novel deoxycytidine analog (gemcitabine) and the topoisomerase I inhibitors (irinotecan and topote- 
can). Optimistic oncologists showed precocious enthusiasm for the efficacy and good toxicity profile as single-agents or in combination with a platinum compounds; as single-agent they produced response rates which exceeded 20\% [12] and 50\% or greater when combined with cisplatin or carboplatin.

When these newer agents plus a platinum ana$\log$ were tested against standard regimens in use at that time platinum-based doublets containing new agents showed improvements in survival in few cases and in most clinical trials proved to be of equivalent efficacy with a better toxicity profile.

Two European studies compared cisplatin/ gemcitabine with either cisplatin/etoposide [13] or mitomycin/ifosfamide/cisplatin (MIC) [14]. Response rates were higher for the gemcitabine/cisplatin regimen: $40 \%$ and $38 \%$ compared to $22 \%$ for etoposide/cisplatin and $26 \%$ for MIC. No differences were noted in 1-year and median survival rates, while in the Spanish study a statistically significant advantage in time to progression was achieved in the gemcitabine arm (6.9 vs 4.3 months).

In this issue Berghmans et al. demonstrated how MIC combination is retaining its activity in an off-protocol setting and the participation in a clinical trail is not ending up in a better survival. This information is reinforcing MIC as a reasonable therapeutic strategy in our daily clinical practice [15].

Results are however conflicting. An optimist could see an evident improvement in response rates (even though not always statistically significant) with newer combinations, which proved to be at least as effective as older ones, with an equivalent if not better toxicity profile and an overall improvement of quality of life. In almost all cases the combination with a platinum derivative is superior to single-agent cisplatin. The negative view can stress the limited survival advantage gained at the expense of sometimes higher toxicity (although usually manageable) and of definitively higher costs compared to older drugs of the 1980s. Moreover, historical comparisons with older regimens are difficult due to stage and prognostic factors migration.

However, the superiority of any newer regimen in terms of improved survival, quality of life, and toxicity profile remains unclear. The more commonly used platinum-based doublets including a newer agent were compared "face to face" in four randomised clinical studies. All these studies have failed to demonstrate the superiority of any one of these so-called third-generation regimens over the others in terms of improved tumour response rates or survival rates, although the regimens do have different toxicity profiles [16].

Given the similarity in the effectiveness of combination regimens, decisions regarding treatment for advanced NSCLC should take into account the toxicity profiles, convenience and costs. Healthcare utilisation data is beginning to be collected prospectively during clinical trials of chemotherapeutic regimens so that economic analyses can be performed.
In conclusion, in advanced non-small cell lung cancer efficacy outcomes of phase III studies of third generation agents plus platinum compounds point in the direction of an "efficacy plateau". Molecular targeted therapies and pharmacogenomic represent reliable platforms for the next generation of clinical trials.

Preclinical research has now made available an array of keys to explain the biologic mechanisms involved in cellular replication and signaling pathways. Novel agents able to target specific steps of the neoplastic transformation and uncontrolled proliferation have already entered the clinical phases and most of them have already failed, alone or in combination with cytotoxic chemotherapy. At the different levels of the signaling pathways receptor-associated tyrosine kinase inhibitors, a variety of monoclonal antibodies directed against a variety of growth factors and specific inhibitors have been more or less extensively investigated with conflicting results. Although some of these targeted agents have already demonstrated cytotoxic activity in the setting of second- and third line therapy of advanced non-small cell lung cancer, when combined with doublets in the first line setting, they have produced disappointing results.

\section{References}

1. Greenlee RT, Murray T, Bolden S, et al. Cancer statistics, 2000. CA Cancer J Clin 2000; 50: 7-33.

2. Ihde DC Chemotherapy of lung cancer. $N$ Engl J Med 1992; 327: 1434-1441.

3. Schroen AT, Detterbeck FC, Crawford R, et al. Beliefs among pulmunologists and thoracic surgeons in the therapeutic approach to non-small cell lung cancer. Chest 2000, 118: 129-137

4. Non-Small Cell Lung Cancer Collaborative Group. Chemotherapy in non-small cell lung cancer: a metaanalysis using updated data on individual patients from 52 randomised clinical trials. BMJ 1995; 311: 899-909.

5. Fernandez C, Rosell R, Abad-Esteve A, et al. Quality of life during chemotherapy in non-small cell lung cancer patients. Acta Oncol 1989; 28: 29-33.

6. Ellis PA, Smith IE, Hardy JR, et al. Symptom relief with MVP (mitomycin C, vinblastine and cisplatin) chemotherapy in advanced non-small-cell lung cancer. Br J Cancer 1995; 71: 366-370.

7. Helsing M, Bergman B, Thaning L, et al. Quality of life and survival in patients with advanced non-small cell lung cancer receiving supportive care plus chemotherapy with carboplatin and etoposide or supportive care only. A multi-centre randomised Phase III trial. Joint Lung Cancer Study Group. Eur J Cancer 1998; 34: 1036-1044.

8. Thongprasert S, Sanguanmitra P, Juthapan W, et al. Relationship between quality of life and clinical outcomes in advanced non-small cell lung cancer: Best supportive care (BSC) versus BSC plus chemotherapy. Lung Cancer 1999; 24: 17-24.

9. Jakkimainen L, Goodwin PJ, Pater J, et al. Counting the costs of chemotherapy in a National Cancer Institute of Canada randomized trial in non small-cell lung cancer. J Clin Oncol 1990; 8: 1301-1309.

10. Smith TJ, Hillner BE, Neighbors DM, et al. Economic evaluation of a randomized clinical trial comparing vinorelbine, vinorelbine plus cisplatin, and vindesine plus cisplatin for non-small-cell lung cancer. J Clin Oncol 1995; 13: 2166- 2173. 
11. Palmer AJ, Brandt A. The cost-effectiveness of four cisplatin-containing chemotherapy regimens in the treatment of stages III B and IV non-small cell lung cancer: an Italian perspective. Monaldi Arch Chest Dis 1996, 51: 279-88.

12. Lilenbaum R, Green MR. Novel chemotherapeutic agents in the treatment of non-small-cell lung cancer. $J$ Clin Oncol 1993; 11: 1391-402.

13. Cardenal F, Lopez-Cabrerizo MP, Anton A, et al. Randomized phase III study of gemcitabine-cisplatin versus etoposide-cisplatin in the treatment of locally advanced or metastatic non-small-cell lung cancer. J Clin Oncol 1999; 17: 12-18.
14. Crino L, Scagliotti GV, Ricci S, et al. Gemcitabine and cisplatin versus mitomycin, ifosfamide, and cisplatin in advanced non-small-cell lung cancer: a randomized phase III study of the Italian Lung Cancer Project. $J$ Clin Oncol 1999; 17: 3522-3530.

15. Berghmans T, Meert AP, Ninane V, Sculier JP. Mitomycin, ifosfamide, cisplatin for non-small cell lung cancer: an implementation study. Monaldi Arch Chest Dis 2005; 63: 184-192.

16. Ettinger DS. Is there a preferred combination chemotherapy regimen for metastatic non-small cell lung cancer? The Oncologist 2002; 7: 226-33.

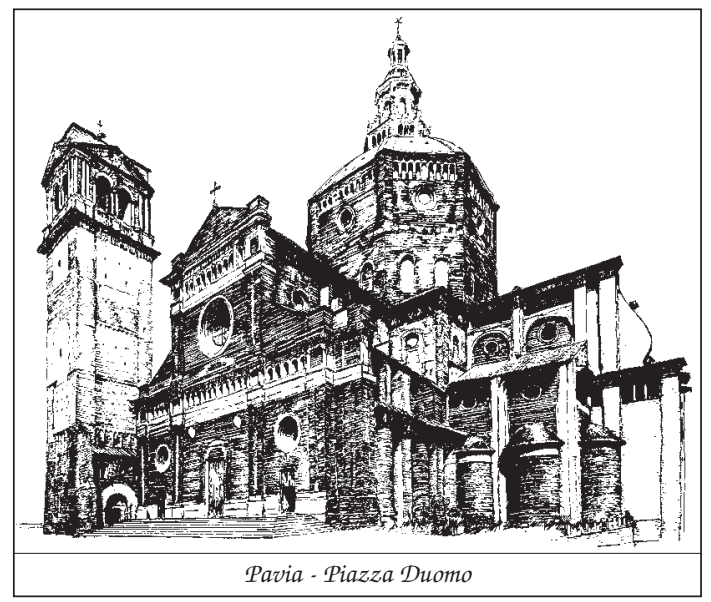

